\title{
Clinical Presentation \& Operative Treatment of Leiomyoma of Uterus and its Outcome
}

Rinku Das ${ }^{1^{*}}$

Rummana Jafrin ${ }^{2}$

Banita Biswas ${ }^{3}$

Nazmun Nahar ${ }^{4}$

Rajat Sanker Roy Biswas ${ }^{5}$

'Department of Gynaecology \& Obstetrics BGC Trust Medical College

Chittagong, Bangladesh.

${ }^{2}$ Department of Gynaecology \& Obstetrics Comilla Medical College

Comilla, Bangladesh.

${ }^{3}$ Department of Gynaecology \& Obstetrics Chittagong Medical College

Chittagong, Bangladesh

${ }^{4}$ Medical Officer

IMAGE, Chittagong

${ }^{5}$ Department of Medicine Chattagram International Dental College

International Islamic Medical College \& Hospital

${ }^{*}$ Correspondence to:

\section{Dr. Rinku Das}

Department of Gynaecology \& Obstetrics BGC Trust Medical College

Chittagong, Bangladesh.

Mobile: +8801713103618

E-mail:dr.rinku123@gmail.com

\begin{abstract}
Introduction: Uterine leiomyoma is remarkably common, however only a subset of women have their fibroids clinically detected, symptomatic, or warrant surgical treatment. Mode of treatment depends on age of the patients, number, size and site of the myoma and also fertility status of the patients. Materials \& methods: To evaluate the sociodemographic, clinical characteristics, management outcome and its determinants in BSMMU, Dhaka, Bangladesh, the study was carried out. 50 patients of diagnosed leiomyoma admitted in the BSMMU, Dhaka was selected for the study. Clinical profile, type of surgery and postoperative follow up findings were noted. Data was analyzed by computer based software SPSS - 18. Results: The common complaints among the 50 patients were progressive menorrhagia (70\%), abdominal swelling (50\%), infertility (24\%), weight in pelvis (38\%), weakness/dizziness or syncopal attack after menstrual period (26\%) and recurrent miscarriages (12\%). The average uterine size at presentation was $15 \pm 9.7$ weeks. The majority $(70 \%)$ of the women presented with multiple leiomyomata. The commonest anatomical position of the myomas were multiple positions and intramural(80\%). Total abdominal hysterectomy with or without bilateral sulphingo ophorectomy according to age was the commonest surgical procedure performed in thirty two (64\%) cases. Myomectomy was done in $12(24 \%)$ cases and laparoscopic assisted vaginal hysterectomy was done in also $6(12 \%)$ of patients. Postoperative complications occurred in $24 \%(12)$ of the women. The most common complications were postoperative pyrexia $12 \%$, blood loss warranting transfusion $8 \%$, wound infection $10 \%$ and prolonged hospital stay $6 \%$. Conclusion: Uterine fibroid is common in our environment and most of the patients had regular menstrual cycle with progressive menorragia. Its removal is commonly associated with post-operative pyrexia, blood loss, and anemia and wound infection. Preoperative correction of anemia appropriate use of antibiotics and strict hemostasis and asepsis can reduce these complications.
\end{abstract}

Key words: Uterine fibroid; leiomyomata; progressive menorrhagia.

\section{INTRODUCTION}

Leiomyoma is the commonest of all pelvic tumors, being present in $20 \%$ of women in the reproductive age group and increasing with age ${ }^{1}$. It is a benign tumor composed mainly of smooth muscle containing varying amount of fibrous tissue. Collagen content of the tumour gives it a heard fibrous texture. The tumor is well circumscribed having a pseudocapsule. Various terms are used to refer the tumor like myoma or fibromyoma and is popularly called fibroid ${ }^{2}$. The cause of uterine leiomyoma is not known. There is evidence that each individual leiomyoma is unicellular in origin (monoclonal) from glucose-6-phosphate dehydrogenase studies ${ }^{3}$. Multiple chromosomal abnormalities are detected in approximately 50 percent of leiomyoma by cytogenetic analysis ${ }^{2}$. Though causes are obscure, oestrogen has been considered as the major promoter of myoma growth. Leiomyoma contain oestrogen receptors in higher concentration than in the surrounding myometrium but in lower concentration than in the endometrium ${ }^{4}$. 
Myoma may arise from any part of mullerian duct, but most commonly develop in the myometrium. The tumor grow slowly, often only manifest in the fourth decade of life. Racial factors have some role in the aetiology of myoma. It is 3-9 times more frequent in black then in white ${ }^{4}$. Leiomyoma is rare before the age of 20 years. They most commonly produce symptoms between the age of 35 to 45 years, but exist in microscopic from before the age of 30 years $^{2}$. The typical leiomyoma is a firm, multinodular structure of variable size, frequently multiple as many as 200 may be found in one uterus. Its rate of to patient, slowing of growth is most likely after menopause, but at least 10 percent of myoma continue to grow after this period $^{5}$. Leiomyoma may be complicated by degeneration, which is most common. Other changes that might occur are in leiomyoma is estimated to be less than 0.2 percent $^{2,5}$. dysmenorrhoea, reduced fertility and recurrent pregnancy $\operatorname{loss}^{4,6}$. In general leiomyoma do not require treatment if it is symptomless and only justifiable to operate on a symptom less tumor when it is larger than 12-14 weeks size of pregnant uterus, if it is growing rapidly, if it is subserous and pedunculated and prone to torsion of its pedicle, if likely to complicate future pregnancy, or if there is doubt about its surgical. Traditionally two main operation are available performed in two to three ways like total abdominal hysterectomy, laporoscopic assisted vaginal hysterectomy and non descendent vaginal hysterectomy(NDVH) $)^{7,8}$. In this short period of study attempts are made to highlight clinical presentation and operative treatment provided to the admitted patients.

\section{MATERIALS \& METHODS}

This was a cross sectional observational study done in the Obstetrics \& Gynaecology department, Bangabandhu Sheikh Mujib Medical University (BSMMU), Dhaka, during a 6 months from Jul 2012 to Dec 2012.Study patients were the all patients admitted in BSMMU, Gynae and Obs. department during the study period who fulfilled the inclusion criteria. Sampling technique were purposive sampling and sample size were 50 patients of leiomyoma. Selection criteria were all patients having leiomyoma admitted in department of Gynaecology and Obstetrics of BSMMU, Dhaka and patients failed medical treatment for leiomyoma and exclusion criteria were pregnancy with leiomyoma of uterus and diagnosis of leiomyoma during Ceserian section. The attendant and/or the patient was thoroughly informed about the aims, objectives and detail procedure of the study before examination. She was encouraged for voluntary participation and allowed freedom to withdraw from the study whenever she liked even after participation. From all eligible subjects clinical history was taken and clinical examination was done. Then treatment procedures of the leiomyoma were monitored and outcomes were also recorded. To determine the anatomical location and number of uterine leiomyoma the intra operative findings recorded in operation notes were used. growth is slow. However, the rate of growth varies from patient torsion, infarction, sarcomatous change etc. Malignant change

Uterine Leiomyoma is a frequent cause of menorrhagia, nature $^{1,2}$. Still now definitive treatment of leiomyoma is myomectomy and hystrectomy. Hystrectomy are usually

All relevant information for each individual study subject was recorded after getting informed written consent on a pretested data sheet. Data were collected by the researcher herself. Data were processed and analyzed by using computer bases software SPSS-18. Different statistical methods were applied for data analysis like percentages and frequency and presented with bar chart and pie chart.

\section{RESULTS}

Regarding analysis of sociodemographic profile it was found that Among the 50 patients $11(22 \%)$ patients were found in the age group $20-29,9(18 \%)$ patients were in age group $30-39$, $20(40 \%)$ patients were found to have age group $40-49$ years, $7(14 \%)$ patients had age group 50-59 years, 3(6\%) patients had age group 60 years and above. Regarding analysis of parity $12(24 \%)$ had parity " 0 ", parity $1-2,3-5,>5$ had $11(22 \%), 23(46 \%)$ and $6(12 \%)$ respectively. Regarding analysis of locality $32(64 \%)$ patients came from urban locality, $10(20 \%)$ patients came from down town and $8(16 \%)$ patients were from rural areas. Among the 50 patients $3(6 \%)$ patient had house hold income less than 5000 taka, 12(24\%) patients had income 5-10,000 taka. 16(32\%) patients had income between $15-20,000$ taka and $6(12 \%)$ patients had income 20 25,000 taka and $3(6 \%)$ had $>25,000$ taka per month. Regarding analysis most of the patients were HSC 22(44\%), 12(24\%) completed SSC, 7(14\%) were below SSC, 5(10\%) were graduate and $4(8 \%)$ were masters.

Table 1: Sociodemographic variables of the study patients

\begin{tabular}{|c|c|c|}
\hline Variables & Number & Percent $(\%)$ \\
\hline \multicolumn{3}{|l|}{ Age } \\
\hline 20-29 years & 11 & $22 \%$ \\
\hline $30-39$ years & 9 & $18 \%$ \\
\hline $40-49$ years & 20 & $40 \%$ \\
\hline $50-59$ years & 7 & $14 \%$ \\
\hline 60 and above & 3 & $6 \%$ \\
\hline \multicolumn{3}{|l|}{ Parity } \\
\hline 0 & 12 & $24 \%$ \\
\hline $1-2$ & 11 & $22 \%$ \\
\hline $3-5$ & 23 & $26 \%$ \\
\hline$>5$ & 6 & $12 \%$ \\
\hline \multicolumn{3}{|l|}{ Residence } \\
\hline Urban & 32 & $64 \%$ \\
\hline Down town & 10 & $20 \%$ \\
\hline Rural & 8 & $16 \%$ \\
\hline \multicolumn{3}{|l|}{ Family income } \\
\hline$<5000$ taka per month & 3 & $6 \%$ \\
\hline $5-10,000$ taka per month & 12 & $24 \%$ \\
\hline $10-15,000$ taka per month & 10 & $20 \%$ \\
\hline $15-20,000$ taka per month & 16 & $32 \%$ \\
\hline $20-25,000$ taka per month & 6 & $12 \%$ \\
\hline$>25,000$ taka per month & 3 & $6 \%$ \\
\hline \multicolumn{3}{|l|}{ Educational status } \\
\hline Below SSC & 7 & $14 \%$ \\
\hline $\mathrm{SSC}$ & 12 & $24 \%$ \\
\hline $\mathrm{HSC}$ & 22 & $44 \%$ \\
\hline Graduate & 8 & $16 \%$ \\
\hline Masters & 4 & $8 \%$ \\
\hline
\end{tabular}


The common complaints among the 50 patients were progressive menorragia (70\%), abdominal swelling (50\%), infertility (24\%), weight in pelvis (38\%), weakness/dizziness or syncopal attack after menstrual period (26\%) and recurrent miscarriages (12\%).

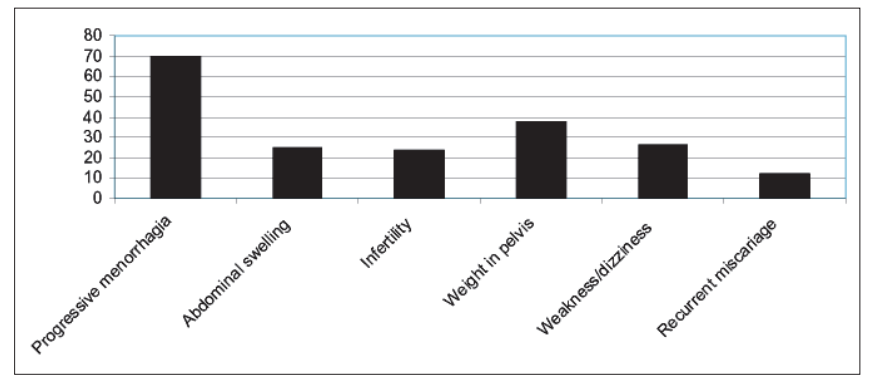

Figure 1: Presenting complaints of the patients

Among the 42 anemic patients 52\% had mild anemia, 29\% had moderate and $19 \%$ had severe anemia.

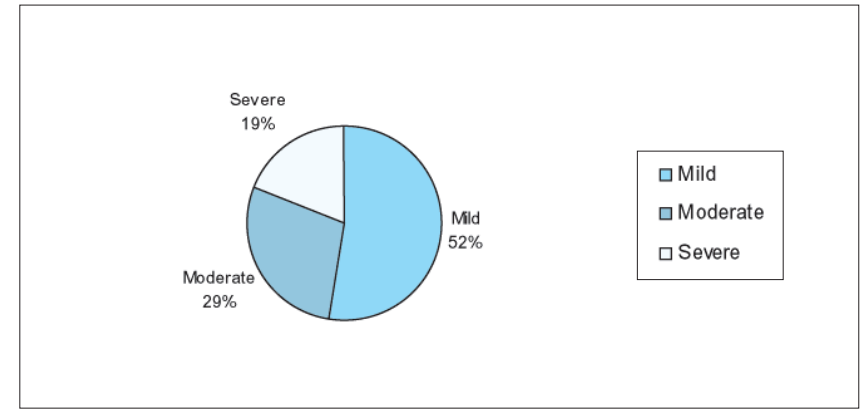

Figure 2: Pattern of anemia

Among 50 women, only 9 (18\%) of cases presented within a year of the onset of symptoms with a mean of $4.9 \pm 4.3$ years. While $24(48 \%)$ women presented between 1-5 years of the onset of symptoms, others presented at 6-10years (20\%) and more than 10years $(8 \%)$. Three women $(6 \%)$ could not recollect exactly the time of onset of the symptoms.

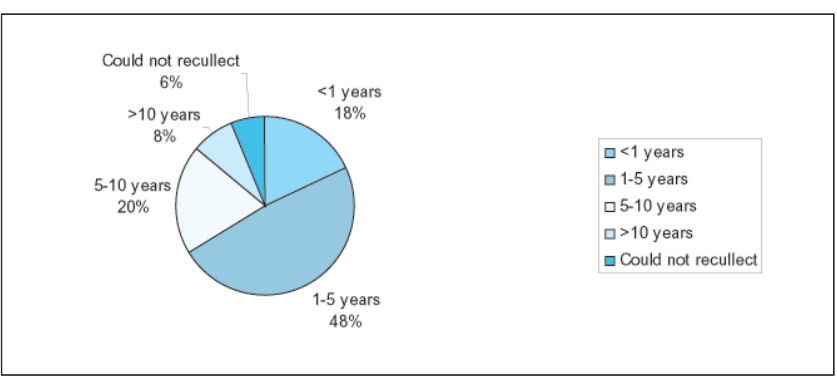

Figure 3 : Duration of presentation after appearance of symptoms

The uterine size at presentation ranged from non-palpable size to size compatible with pregnancy size of 36 weeks with a mean of $15 \pm 9.7$ weeks uterine size. The uterine size was not palpable $20 \%$ ( 8-10 weeks size $8 \%, 10-12$ weeks size $12 \%$ ) 12 14 weeks size $12 \%, 14-16$ weeks size $32 \%, 16-18$ weeks size $12 \%, 18-20$ weeks size- $10 \%$ and $>20$ weeks size was $14 \%$ patients.
Table 2: Size of the uterus

\begin{tabular}{lcc} 
Size & Number & Percent \\
Not palpable & & $20 \%$ \\
8-10 weeks size & 4 & $8 \%$ \\
10-12 weeks size & 6 & $12 \%$ \\
Palpable & & $80 \%$ \\
12-14 weeks size & 6 & $12 \%$ \\
14-16 weeks size & 16 & $32 \%$ \\
$16-18$ weeks size & 6 & $12 \%$ \\
$18-20$ weeks size & 5 & $10 \%$ \\
$>20$ weeks & 7 & $14 \%$ \\
\hline
\end{tabular}

To determine the anatomical location and number of uterine leiomyoma nodules, the intraoperative findings recorded in the operation notes were used. Of the 50 cases managed, while $35(70 \%)$ had multiple uterine leiomyoma numbering 2 to 13 with a mean of $7 \pm 4.3$ myoma, in the remaining 15 (30\%) cases single uterine leiomyoma was the finding. Among the 50 patients $80 \%$ myomas were intramural, $12 \%$ were subserous and $8 \%$ were submucous in type. Among the 50 patients associated pathology like cystic ovary, endometriosis and pelvic inflammatory disease was present among $26 \%$ of patients.

Table 3: Intra operative findings

\begin{tabular}{lccc} 
Size & Number & Percent (\%) \\
$\begin{array}{l}\text { Intra operative findings } \\
\text { (Number of myoma) }\end{array}$ & & & \\
Single & 15 & $30 \%$ & \\
Multiple & 35 & $70 \%$ & $80 \%$ \\
Type of leiomyoma & & & \\
Intramural & 40 & $80 \%$ & \\
Subserus & 6 & $12 \%$ & \\
Submucus & 4 & $8 \%$ & \\
Associated pathology & & & \\
& & & \\
Present & 13 & $26 \%$ & \\
Absent & 37 & $74 \%$ \\
\hline
\end{tabular}

The surgery was performed by the consultant staffs . The skin incision for the entry of the abdomen was Midline and Pfannenstiel in $46(92 \%)$ and $4(8 \%)$ respectively. Total abdominal hysterectomy with or without bilateral sulphingo ophorectomy according to age was the commonest surgical procedure performed in thirty two(64\%) cases. Myomectomy was done in $12(24 \%)$ cases and laparoscopic assited vaginal hysterectomy was done in also 6(12\%) of patients.

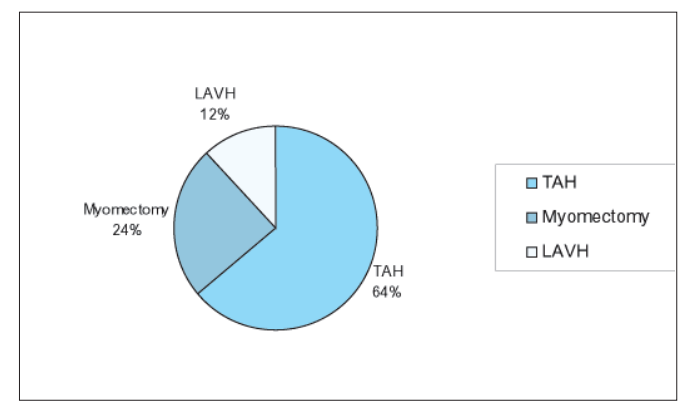

Figure 4: Types of operation 
Postoperative complications occurred in 12(24\%) women. The most common complications were postoperative pyrexia $6(12 \%)$, blood loss warranting transfusion $4(8 \%)$, wound infection 5(10\%) and prolonged hospital stay $3(6) \%$.

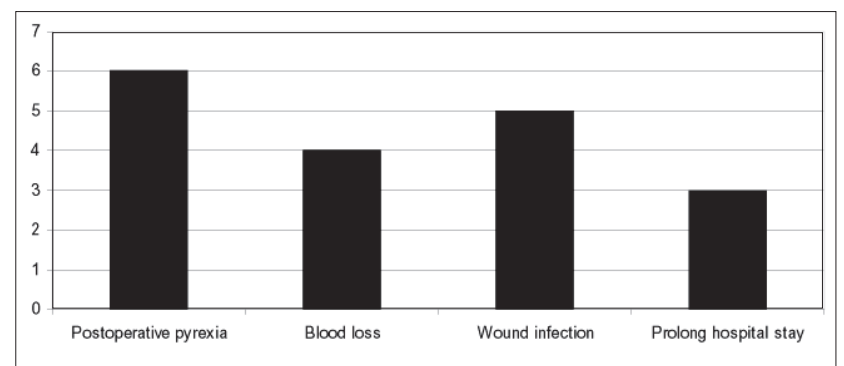

Figure 5: Postoperative complications

\section{DISCUSSION}

Uterine leiomyoma are the most common female reproductive tract tumour, however majority of cases are asymptomatic. Several studies have documented an increased incidence of uterine leiomyoma in black women and women of African descent. In a study it was found that $9.3 \%$ of all gynaecological cases were the uterine leiomyoma? ${ }^{7}$. The finding is similar to $9.8 \%$ reported from Lokoja Nigeria, but higher than $7.8 \%$ and $8.4 \%$ reported from two Nigerian cities of Zaria and Ilesa respectively. As the majority of the uterine fibroids are symptomless, the incidence remains speculative In a study that utilized ultrasound scan to screen women for leiomyoma, the prevalence ranged from $4 \%$ in women 20 to 30 years of age to $14.5 \%$ in women aged 30 to 40 years of age and $33 \%$ in women 40 to 60 years of age ${ }^{8}$.

The present study was done in the Gynae and Obstretics Department of BSMMU where 50 patients were included for treatment for the diagnose case of leiomyoma. Peak age of presentation was 30 to 49 years. In the Caucasians population uterine leiomyoma tend to occur around the age of 30years and commonly causes symptoms between ages 35 and 45 years. In a review, majority, $837(72.1 \%)$ of the cases occurred in the third and fourth decades of life; in keeping with similar reports from our environrnent. The reasons for the high incidence of fibromyoma as from third decades is highly speculative, however female sex hormones have been implicated, particularly stimulation by oestrogen unbalanced by progesterone as a result of persistent anovulation. Nulliparous and primparous women accounted for $44.4 \%$ of cases in this review in keeping with observations that leiomyoma are common in the nulliparous or relatively infertile women.

Most of the patients were from urban location as BSMMU is located in the capital city and access to it to rural community is not easy. Most of the family of the patients had middleclass socioeconomic status and their education background were around HSC level. As most of the female came from the urban area these educational and socioeconomic status did not match the actual scenario of our community.
The commonest symptoms of presentation of the leiomyoma was progressive menorragia(70\%) and abdominal swelling $(50 \%)$. Attributing symptoms specifically to uterine fibroid is somewhat problematic because of its high incidence in the population, variable clinical presentation and often asymptomatic nature. However evidence largely drawn from uncontrolled studies, showed that uterine fibroid are commonly identified in women who have menorrhagia, abdominal lump, infertility, or recurrent pregnancy loss. Abnormal uterine bleeding was the commonest presenting symptoms in these women with confirmed uterine fibroid ${ }^{9}$.

The cause of the increased uterine bleeding is not always clear but have been variously been attributed to increase surface area and hyperplasia of endometrium, increased vascularity of the uterus, interstitial fibroid nodules that prevent myometrial contraction and functional ovaries often found in association with uterine fibroid. However a population based study did not find any evidence relating general abnormalities in menstrual cycle length or heaviness to the presence of uterine fibroids ${ }^{10}$.

There is a well-recognized association between uterine fibroid and infertility; however the actual contribution of fibroid in infertility remains controversial. Many of the studies examining relationship between fibroid and infertility are retrospective and non-randomised. Current evidence suggests that submucosal and intramural fibroids that distort the uterine cavity can impair in vitro fertilization attempts. The impact of intramural and subserosal fibroids that do not distort the intrauterine cavity is unclear. Despite the lack of clear evidence of their role in preventing conception, submucosal fibroid, intramural fibroids that distort the uterine cavity, fibroids larger than $5 \mathrm{~cm}$, and multiple fibroids are often treated in patients with otherwise unexplained infertility. However one fact is clear, infertility either voluntary or involuntary is likely to be followed in time with the development of uterine fibroid, and on the other hand, once fibroid have developed, fertility is likely to be decrease or in abeyance $e^{11,12}$.

Total abdominal hysterectomy with or without salphingo ophorectomy was the commonest (64\%) treatment option among the present study patients at BSMMU. Myomectomy and laporescopic assisted vaginal hysterectomy were also performed in some patients. In a study done in abroad myomectomy was the commonest surgery performed, accounting for $54.7 \%$ of cases. The remaining had hysterectomy $(43.8 \%)$ or polypectomy alone $(1.5 \%)$. Female presented with the low parity, presenting with infertility and recurrent miscarriage, myomectomy being the commoner surgical procedure is expected. Myomectomy was often done in patients with associated infertility to improve their fertility potential or when the fibroid is asymptomatic in women who have not completed their family. 
Hysterectomy is usually performed in women who had symptomatic fibroids and had completed their family. The decision to retain the ovaries often depends on how far or close a patient is to menopause and presence of any disease condition involving ovaries.

The postoperative complication ranged from post operative pyrexia (12\%), blood loss warranting blood transfusion $(8 \%)$, wound infection $(10 \%)$, and prolonged hospital stay (3\%). Its heart warming that majority of the patients did not have complications. Post operative pyrexia is a common feature of surgical practice in the tropics. This may be due to wound infection or as a result of oozing of blood into the myomectomy and peritoneal cavity.

Myomectomy is generally associated with higher morbidity than hysterectomy, not only that it is technically more difficult but associated with longer operation time, greater tissue injury and blood loss.
If proper asepsis and hemostasis is maintained peroperative and postoperative complications can be avoided ${ }^{13}$. With increased number of fibroid nodes, surgery is likely to be prolonged with increased tissue injury, blood loss and surgeon fatigue. These are factors known to be associated increased postoperative morbidity.

\section{CONCLUSION}

Uterine fibroid is common in our country and usually patients present with menorrhagia, pelvic lump, recurrent miscarriage and infertility. If patients present early some complications like severe anemia, recurrent miscarriage can be prevented. In young patient uterus can be preserved if they present earlier.

\section{DISCLOSURE}

All the authors declared no competing interest. 


\section{REFERENCES}

1. Dewhurst's Textbook of Obsterics and Gynaecology for post-graduates. Edited by D. Keith Edmonds. Edition Blackwell Science. Benign tumor of uterus. 2007; 552-558.

2. Tindal VR ed: Jeffcoate principles of Gynaecology ( $7^{\text {th }}$ ed) London Butter Worth \& Co. 2011;20-38, 68-87, 467-482.

3. Current obsetetries and gynaecologic diagnosis and treatment $10^{\text {th }}$ ed. 2006: 731-740.

4. Cramer, SF, \& Patel, A. . The frequency of uterine leiomyomas. American Journal of Clinical Pathology,2010; 94: 435-438.

5. Pritts, EA.. Fibroids and infertility: A systemic review of the evidence. Obstet Gynecol Surv, Aug; 2001; 58(8): 483-491.

6. Women's Health and the Environment: The Next Century - Advances in Uterine Leiomyoma Research, Conference Proceedings, . Environ health Perspec, 2006; 108: (suppl 5), 767-853.

7. Ezem BU, Otubu JA. Hysterectomy in the Hausa/Fulani population in Nigeria. International journal of Gynecology and Obstetrics. 1981;19:145-147.

8. Ogunniyi SO, Fasubaa OB. Uterine Fibromyoma in Ilesha, Nigeria. Nigerian Medical Practioner. 1990;19(6):93-95.

9. Jonas HS, Masterdon BJ. Giant uterine tumours: case report and review of literature. Obstetrics and Gynecology. 1972;50:25-29.

10. Omu AE, Ehiegiegba EA. Myomectomy: a reappraisal of postoperative performance. Asia and Oceania Journal of Obstetrics and Gynaecology. 1983;9(1):43-48.

11. Akinola OI, Ottun TA, Fabanwo RO, Akinniyi OA. Blateral uterine artery ligation: an effective low technology option in the management of symptomatic uterine fibroids. Tropical Journal of Obstetrics and Gynaecology. 2003;20:4-6.

12. Witherspoon JT. The hormonal origin of uterine fibroid: a hypothesis. American Journal of Cancer. 1935;24:402-406.

13. Bajekal N, Li TC. Fibroids, infertility and pregnancy wastage. Human Reproduction Update. 2000;6:614-620. 